\title{
Trajectory tracking for omnidirectional mecanum robot with longitudinal slipping
}

\author{
Renhui Zhang ${ }^{1}$, Haiyan $\mathrm{Hu}^{1}$ and Yongsi $\mathrm{Fu}^{1}$ \\ ${ }^{1}$ School of Mechanical and Electric Engineering, Soochow University, 215006, Suzhou, China
}

\begin{abstract}
This paper propose an adaptive control law for an omnidirectional mecanum robot under longitudinal slip conditions. Firstly, the control law under pure rolling is obtained on the base of Lyapunov function, and the stability of the close-loop is proved. Secondly, four unknown parameters are used to describe the wheel slip coefficient, and the tracking error differential equations under longitudinal slip are established. Then, an adaptive nonlinear feedback controller is constructed, and using an appropriate Lyapunov function to ensure the close-loop system is stable. Matlab/Simulation result confirms the effectiveness of the proposed approach.
\end{abstract}

\section{Introduction}

Because of the characteristic of non-holonomic constraint, the research for control of mobile robots has drawn much attention. Many researchers have designed a tracking and stabilization controller for non-holonomic mobile robots with nonlinear control methods, such as back-stepping control[1-4], sliding mode control[5-6], adaptive control[7-8], fuzzy control[9-11], and intelligent control based on neural networks[12-13].

Majority of previous literatures[1-13] assume that the controlled wheeled mobile robot satisfies the assumption of pure rolling non-holonomic constraints. However, in practical, wheels will inevitably slide due to wet ground, rapid turning, etc. And destroying the nonholonomic constraints to overcome this problem, it is necessary to study the mobile robot control method that considers the slip factor[14-17]. Juliano $G$ et al.[14,15] use two unknown parameters describe slip of the left and right wheel. Gonzalez R et al.[16] use two feedback gain for trajectory tracking of robots under slipping. Endo D et al.[17] propose a method of odometry estimates the slip ratios using encoders and gyro-sensor. Mecanum wheel mobile platform relies on the different rotational speeds of the wheels to achieve omnidirectional movement, so the movement of each wheel contributes a lot to the overall direction of motion and speed. If the wheel is slipping, the movement direction of the entire mobile platform will deviate. Therefore, the longitudinal slip of the Mecanum wheel will lead to instability of the motion platform. However there is little research of trajectory tracking for Omni-directional Mecanum Robot (ODMR) with longitudinal slipping.

The main achievement of this paper is designing an adaptive control law for tracking desire trajectory of ODMR under longitudinal slip conditions based on Lyapunov function.

\section{Kinematics on mecanum wheel robot}

This article begins with the derivation of the inverse kinematics equation for the robot. Due to the differences between Mecanum wheel and the standard wheel, the algorithms required to control the robot's motion are very different from controlling differential drive robot.

Figure 1 shows the schematic model of ODMR, the robot coordinate attached to the frame, wheel coordinate attached to the wheel. All of the rollers have the same tilted angles which are $\pm 45^{\circ}$, and each roller can rotate around its axes. Figure 2 is the wheel 1 coordinate in robot coordinate.
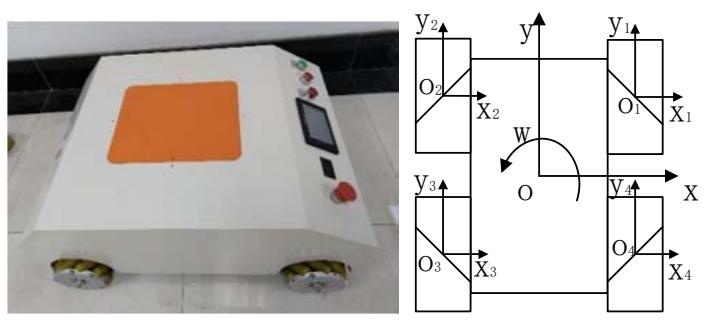

Fig. 1. Omnidirectional mecanum wheel robot.
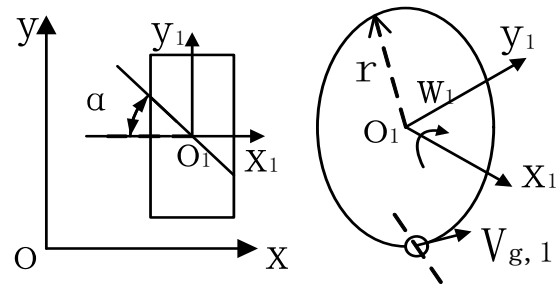

Fig. 2. Wheel 1 coordinate. 
The robot parameters are defined as follows:

$r$ is radius of wheel, $w_{i}$ is angular velocity of No.i wheel, $\alpha_{i}$ is tilted angles of rollers of No.i wheel, $v_{g, i}$ is angular velocity of rollers of No.i wheel, $l_{x}$ is the half distance in the x-axis of two wheels, $l_{y}$ is the half distance in the $\mathrm{y}$-axis of two wheels, $\left[\begin{array}{lll}x_{i} & y_{i} & \alpha_{i}\end{array}\right]^{T}$ is the parameter of No.i wheel, $\left[\begin{array}{lll}v_{x} & v_{y} & w\end{array}\right]^{T}$ is the centroid velocity of robot.

Analyze the velocity of a wheel in Robot Coordinate, the velocity of a wheel in Wheel Coordinate and centroid velocity of the robot in Robot Coordinate.

We obtain:

$$
\begin{gathered}
{\left[\begin{array}{c}
v_{i, x} \\
v_{i, y}
\end{array}\right]=\left[\begin{array}{cc}
0 & -\sin \alpha_{i} \\
r & \cos \alpha_{i}
\end{array}\right]\left[\begin{array}{c}
w_{i} \\
v_{g, i}
\end{array}\right]=\left[\begin{array}{ccc}
1 & 0 & -y_{i} \\
0 & 1 & x_{i}
\end{array}\right]\left[\begin{array}{l}
v_{x} \\
v_{y} \\
w
\end{array}\right]} \\
w_{i}=\frac{1}{r}\left[\frac{1}{\tan \alpha_{i}} 11 x_{i}-\frac{y_{i}}{\tan \alpha_{i}}\right]\left[\begin{array}{c}
v_{x} \\
v_{y} \\
w
\end{array}\right]
\end{gathered}
$$

The Jacobian matrix $J$ of omnidirectional mecanum mobile robot shown in Equation (3).

$$
J=\frac{r}{4}\left[\begin{array}{cccc}
-1 & 1 & -1 & 1 \\
1 & 1 & 1 & 1 \\
\frac{1}{l_{x}+l_{y}} & \frac{1}{l_{x}+l_{y}} & \frac{1}{l_{x}+l_{y}} & \frac{1}{l_{x}+l_{y}}
\end{array}\right]
$$

The forward of the ODMR are given in Equation (4).

$$
\left[\begin{array}{c}
v_{x} \\
v_{y} \\
w
\end{array}\right]=J\left[\begin{array}{l}
w_{1} \\
w_{2} \\
w_{3} \\
w_{4}
\end{array}\right]
$$

The data of the robot and the wheel are listed in Table 1.

Table 1. Parameterization of the robot

\begin{tabular}{|c|c|c|}
\hline Parameter & length & unit \\
\hline Diameter of wheel & 127 & $\mathrm{~mm}$ \\
\hline Length of $l_{x}$ & 267 & $\mathrm{~mm}$ \\
\hline Length of $l_{y}$ & 267 & $\mathrm{~mm}$ \\
\hline
\end{tabular}

\section{Tracking control law for ODMR without slipping}

For the tracking problem, it is mean that at any time, find an auxiliary input $\eta=\left[\begin{array}{lllll}w_{1} & w_{2} & w_{3} & w_{4}\end{array}\right]^{T}$, such that $\lim _{t \rightarrow \infty}\|E\|=0$, where $E$ is defined as the trajectory tracking error:

$$
E=\left[\begin{array}{l}
x_{e} \\
y_{e} \\
\theta_{e}
\end{array}\right]=\left[\begin{array}{ccc}
\cos \theta & \sin \theta & 0 \\
-\sin \theta & \cos \theta & 0 \\
0 & 0 & 1
\end{array}\right]\left[\begin{array}{l}
x_{r}-x \\
y_{r}-y \\
\theta_{r}-\theta
\end{array}\right]
$$

Where $q_{r}=\left[\begin{array}{lll}x_{r} & y_{r} & \theta_{r}\end{array}\right]^{T}$ is the reference trajectory, $q=\left[\begin{array}{lll}x & y & \theta\end{array}\right]^{T}$ is coordinate of the robot.

The differential equation of tracking error is described by the equations (6).

$$
E^{\prime}=\left[\begin{array}{l}
x_{e}^{\prime} \\
y_{e}^{\prime} \\
\theta_{e}^{\prime}
\end{array}\right]=\left[\begin{array}{ccc}
-1 & 0 & y_{e} \\
0 & -1 & -x_{e} \\
0 & 0 & -1
\end{array}\right]\left[\begin{array}{l}
v_{x} \\
v_{y} \\
w
\end{array}\right]+\left[\begin{array}{c}
v_{x, r} \\
v_{y, r} \\
w_{r}
\end{array}\right]
$$

A Lyapunov function can be selected as follow:

$$
V_{1}=\frac{1}{2} x_{e}^{2}+\frac{1}{2} y_{e}^{2}+\sin ^{2} \frac{1}{2} \theta_{e} \geq 0
$$

The derivative of $V_{1}$ can be described as:

$$
V_{1}^{\prime}=x_{e}\left(v_{x, r}-v_{x}\right)+y_{e}\left(v_{y, r}-v_{y}\right)+\frac{1}{2} \sin \theta_{e} *\left(w_{r}-w\right)
$$

Choose trajectory tracking control laws Equation (9).

$$
\begin{gathered}
\left\{\begin{array}{lc}
v_{x}=v_{x, r}+C_{1} x_{e} & C_{1}>0 \\
v_{y}=v_{y, r}+C_{2} y_{e} & C_{2}>0 \\
w=w_{r}+C_{3} \sin \theta_{e} & C_{3}>0
\end{array}\right. \\
V_{1}^{\prime}=-C_{1} x_{e}^{2}-C_{2} y_{e}^{2}-C_{3} \sin ^{2} \theta_{e} \leq 0
\end{gathered}
$$

It shows $\lim _{t \rightarrow \infty}\|E\|=0$ by Lyapunov function.

\section{Adaptive control law longitudinal slipping}

Define the longitudinal slip ratio of wheels as follows:

$$
s=\frac{r w-v}{r w} \in[0,1)
$$

Where $v$ is the linear velocity of the wheel, $r$ is the radius of the wheel, $w$ is the angular velocity of the wheel.

However, longitudinal slip ratio is difficult to precisely measure the $\operatorname{slip}[14]$. Assume that $\hat{S}$ is approximating of longitudinal slip ratio matrix $S$.

$$
S=\left[\begin{array}{cccc}
s_{1} & 0 & 0 & 0 \\
0 & S_{2} & 0 & 0 \\
0 & 0 & s_{3} & 0 \\
0 & 0 & 0 & s_{4}
\end{array}\right] \hat{S}=\left[\begin{array}{cccc}
\hat{S}_{1} & 0 & 0 & 0 \\
0 & \hat{s}_{2} & 0 & 0 \\
0 & 0 & \hat{s}_{3} & 0 \\
0 & 0 & 0 & \hat{s}_{4}
\end{array}\right]
$$

In this paper, it is assumed that the wheels are only affected by the longitudinal slip, the actual velocity under longitudinal slip can be described by the following Equation (13).

$$
V_{\text {slip }}=\left[\begin{array}{c}
v_{x, \text { slip }} \\
v_{y, \text { slip }} \\
w_{\text {slip }}
\end{array}\right]=J(E-S)(E-\hat{S})^{-1} J^{-1}\left[\begin{array}{c}
v_{x} \\
v_{y} \\
w
\end{array}\right]
$$

Where $\left[\begin{array}{lll}v_{x} & v_{y} & w\end{array}\right]^{T}$ is given by trajectory tracking control laws.

Redefine slipping parameter to simplify the calculation.

$$
q_{k}=\frac{1}{1-s_{k}}, \hat{q}_{k}=\frac{1}{1-\hat{s}_{k}}, \tilde{q}_{k}=\hat{q}_{k}-q_{k}
$$

Where $\tilde{q}_{k}$ is the estimation error of $q_{k}$. 
The differential equation of tracking error under the slow transformation of the longitudinal slip parameters become:

$$
\begin{gathered}
A=\left[\begin{array}{cccc}
-\left(\frac{1}{4}+\frac{y_{e}}{4 a}\right) & -\left(\frac{1}{4}+\frac{y_{e}}{4 a}\right) & -\left(\frac{1}{4}-\frac{y_{e}}{4 a}\right) & -\left(\frac{1}{4}-\frac{y_{e}}{4 a}\right) \\
\left(\frac{1}{4}+\frac{x_{e}}{4 a}\right) & -\left(\frac{1}{4}-\frac{x_{e}}{4 a}\right) & \left(\frac{1}{4}-\frac{x_{e}}{4 a}\right) & -\left(\frac{1}{4}+\frac{x_{e}}{4 a}\right) \\
\frac{1}{4 a} & \frac{1}{4 a} & -\frac{1}{4 a} & -\frac{1}{4 a}
\end{array}\right] \\
B=\left[\begin{array}{lll}
\frac{\tilde{q}_{1}}{q_{1}} & -\frac{\tilde{q}_{1}}{q_{1}} & -a \frac{\tilde{q}_{1}}{q_{1}} \\
\frac{\tilde{q}_{2}}{q_{2}} & \frac{\tilde{q}_{2}}{q_{2}} & -a \frac{\tilde{q}_{2}}{q_{2}} \\
\frac{\tilde{q}_{3}}{q_{3}} & -\frac{\tilde{q}_{3}}{q_{3}} & a \frac{\tilde{q}_{3}}{q_{3}} \\
\frac{\tilde{q}_{4}}{q_{4}} & \frac{\tilde{q}_{4}}{q_{4}} & a \frac{\tilde{q}_{4}}{q_{4}}
\end{array}\right] \\
E_{s l i p}^{\prime}=E^{\prime}+A B\left[\begin{array}{c}
v_{x} \\
v_{y} \\
w
\end{array}\right]
\end{gathered}
$$

Where $a=l_{x}+l_{y}$.

A Lyapunov function can be selected as follows:

$$
\begin{aligned}
& V_{2}=\frac{1}{2} x_{e}^{2}+\frac{1}{2} y_{e}^{2}+\sin ^{2} \frac{1}{2} \theta_{e} \\
& +\frac{\tilde{q}_{1}^{2}}{2 \rho_{1} q_{1}}+\frac{\tilde{q}_{2}^{2}}{2 \rho_{2} q_{2}}+\frac{\tilde{q}_{3}^{2}}{2 \rho_{3} q_{3}}+\frac{\tilde{q}_{4}^{2}}{2 \rho_{4} q_{4}} \geq 0
\end{aligned}
$$

Where $\rho_{1}, \rho_{2}, \rho_{3}, \rho_{4}>0$.

$$
\begin{gathered}
V_{2}{ }^{\prime}=V_{1}{ }^{\prime}+\frac{\tilde{q}_{1}}{4 q_{1}} Q_{1}+\frac{\tilde{q}_{2}}{4 q_{2}} Q_{2}+\frac{\tilde{q}_{3}}{4 q_{3}} Q_{3}+\frac{\tilde{q}_{4}}{4 q_{4}} Q_{4} \\
C=\left[\begin{array}{ccc}
-v_{x}+v_{y}+a w & v_{x}-v_{y}-a w & v_{x}-v_{y}-a w \\
-v_{x}-v_{y}+a w & -v_{x}-v_{y}+a w & v_{x}+v_{y}-a w \\
-v_{x}+v_{y}-a w & v_{x}-v_{y}+a w & -v_{x}+v_{y}-a w \\
-v_{x}-v_{y}-a w & -v_{x}-v_{y}-a w & -v_{x}-v_{y}-a w
\end{array}\right]
\end{gathered}
$$

$$
\left[\begin{array}{c}
Q_{1} \\
Q_{2} \\
Q_{3} \\
Q_{4}
\end{array}\right]=C\left[\begin{array}{c}
x_{e} \\
y_{e} \\
\frac{1}{2 a} \sin \theta_{e}
\end{array}\right]++\left[\begin{array}{c}
\frac{4 \hat{q}_{1}^{\prime}}{\rho_{1}} \\
\frac{4 \hat{q}_{2}{ }^{\prime}}{\rho_{2}} \\
\frac{4 \hat{q}_{3}^{\prime}}{\rho_{3}} \\
\frac{4 \hat{q}_{4}^{\prime}}{\rho_{4}}
\end{array}\right]
$$

Choosing update rule for $\hat{q}_{1}, \hat{q}_{2}, \hat{q}_{3}, \hat{q}_{4}$ shown in Equation (22).

$$
\left[\begin{array}{l}
\hat{q}_{1}{ }^{\prime} \\
\hat{q}_{2}{ }^{\prime} \\
\hat{q}_{3}{ }^{\prime} \\
\hat{q}_{4}{ }^{\prime}
\end{array}\right]=\left[\begin{array}{cccc}
-\frac{\rho_{1}}{4} & 0 & 0 & 0 \\
0 & -\frac{\rho_{2}}{4} & 0 & 0 \\
0 & 0 & -\frac{\rho_{3}}{4} & 0 \\
0 & 0 & 0 & -\frac{\rho_{4}}{4}
\end{array}\right] C\left[\begin{array}{c}
x_{e} \\
y_{e} \\
\frac{1}{2 a} \sin \theta_{e}
\end{array}\right]
$$

$$
\Rightarrow V_{2}{ }^{\prime}=V_{1}{ }^{\prime} \leq 0
$$

Figure 3 shows the schematic diagram of the closeloop system composed of the adaptive kinematic controller, the reference trajectory and the robot.

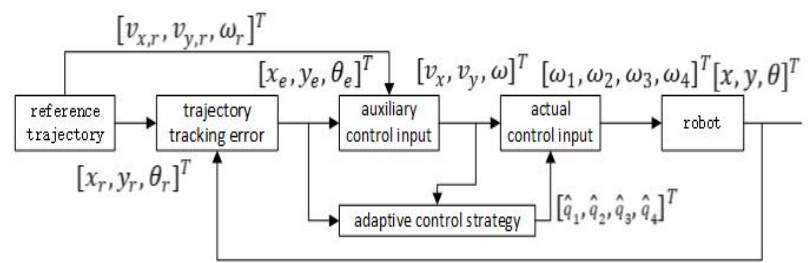

Fig. 3. Schematic diagram of the close-loop system.

\section{Conclusion}

The physical parameters of the ODMR, take from table1, are $r=0.0635 m$ and $l_{x}+l_{y}=0.267 \mathrm{~m}$, the parameters of the tracking control law are chosen as $C_{1}=C_{2}=1, C_{3}=5$ and the parameters of the update longitudinal slipping rule are chosen as $\rho_{1}=1, \rho_{2}=$ $1, \rho_{3}=1, \rho_{4}=1$.

The initial state of robot is set to be $q(0)=\left[\begin{array}{lll}-2 & 1 & 1\end{array}\right]^{T}$, the reference inputs is given like Equation (24).

$$
\left\{\begin{array}{l}
x_{r}(t)=0.5 t \\
y_{r}(t)=\sin (t)+0.1 t \\
\theta_{r}(t)=0.2 t
\end{array}\right.
$$
(25).

The Slip ratio parameter inputs shown in Equation

$$
\begin{aligned}
& q_{1}= \begin{cases}1, & 0 \leq t \leq 5 \\
2, & t \geq 5\end{cases} \\
& q_{2}= \begin{cases}2, & 0 \leq t \leq 5 \\
3, & t \geq 5\end{cases} \\
& q_{3}= \begin{cases}2.5, & 0 \leq t \leq 5 \\
1, & t \geq 5\end{cases} \\
& q_{4}= \begin{cases}3, & 0 \leq t \leq 5 \\
1.2, & t \geq 5\end{cases}
\end{aligned}
$$

We can get the simulation result of the system as follows:

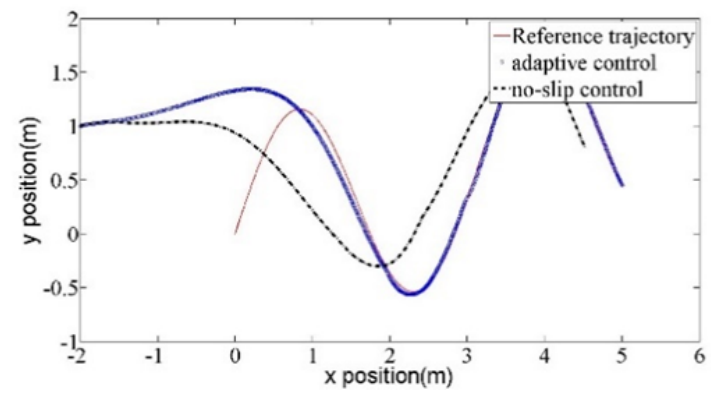

Fig. 4. Trajectory of the mobile robot. 


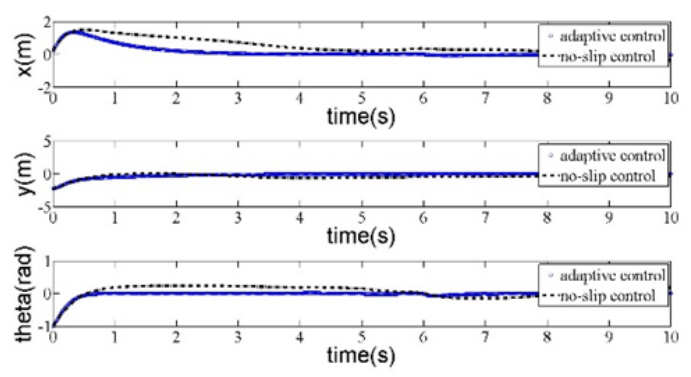

Fig. 5. Trajectory tracking error.

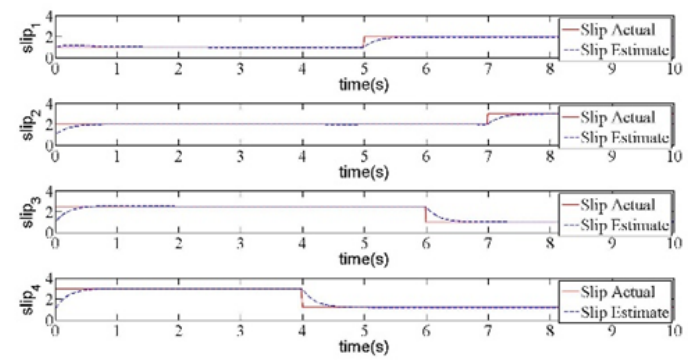

Fig. 6. Slip ratio of wheels.

Figure 4, Figure 5 and Figure 6 show that the adaptive control law which is consisted of the slip rate updating law and auxiliary control law can drive tracking error of mobile robot to 0 under the longitudinal slipping, but the control law without longitudinal slip can't.

The simulation results indicate that the adaptive control law proposed in this paper has better tracking performance than the control law without longitudinal slip.

\section{References}

1. Jiangdagger Z P, Nijmeijer H. Tracking Control of Mobile Robots: A Case Study in Backstepping. Automatica, 33.7(1997):1393-1399.

2. Nganga-Kouya D, Okou F A. Adaptive backstepping control of a wheeled mobile robot. Control and Automation (2009):85-91.

3. Wilson, D. G., and I. I. I. Robinett. Robust adaptive backstepping control for a nonholonomic mobile robot. IEEE International Conference on Systems, Man, and Cybernetics (2002):3241-3245 vol.5.

4. Hao Y, Wang J, Chepinskiy S A, et al. Backstepping Based Trajectory Tracking Control for a FourWheel Mobile Robot with Differential-Drive Steering. Chinese Control Conference (2017):49184923.

5. Zhang J, Zhou B, Wei S, et al. Study on sliding mode trajectory tracking control of mobile robot based on the Kalman filter. IEEE International Conference on Information and Automation (2017):1195-1199.
6. Bessas A, Benalia A, Boudjema F. Integral Sliding Mode Control for Trajectory Tracking of Wheeled Mobile Robot in Presence of Uncertainties. J. Control Sci. Eng, 2016.3(2016):1-10.

7. Alakshendra V, Chiddarwar S S. Adaptive robust control of Mecanum-wheeled mobile robot with uncertainties. Nonlinear Dynamics, 87.4(2017):2147-2169.

8. Alakshendra V, Chiddarwar S S. A robust adaptive control of mecanum wheel mobile robot: simulation and experimental validation. Ieee/rsj International Conference on Intelligent Robots and Systems. (2016):5606-5611.

9. Akka K, Khaber F. Optimal tracking control of a trajectory planned via fuzzy reactive approach for an autonomous mobile robot. Int. J. Adv. Robot. Syst. 15.1(2018):230-8.

10. Al-Dahhan M R H, Ali M M. Path tracking control of a mobile robot using fuzzy logic. International Multi-Conference on Systems, Signals \& Devices (2016):82-88.

11. Fahmizal, Kuo C H. Trajectory and heading tracking of a mecanum wheeled robot using fuzzy logic control. International Conference on Instrumentation, Control and Automation (2017).

12. Tsai C C, Wu H L, Tai F C. Intelligent sliding-mode formation control for uncertain networked heterogeneous Mecanum-wheeled omnidirectional platforms. IEEE International Conference on Systems, Man, and Cybernetics (2017):000539000544.

13. Yang $\mathrm{C}$, Teng $\mathrm{T}, \mathrm{Xu} \mathrm{B}$, et al. Global adaptive tracking control of robot manipulators using neural networks with finite-time learning convergence. Int. J. Control Autom. Syst. 15.11(2017):1-9.

14. Iossaqui J G, Camino J F, Zampieri D E. A Nonlinear Control Design for Tracked Robots with Longitudinal Slip. IFAC Proc. Vol. 44.1(2011):5932-5937.

15. Iossaqui J G, Camino J F. Wheeled robot slip compensation for trajectory tracking control problem with time-varying reference input. Robot Motion and Control (2013):167-173.

16. Gonzalez R, Fiacchini M, Alamo T, et al. Adaptive Control for a Mobile Robot Under Slip Conditions Using an LMI-Based Approach. Eur J Control, 16.2(2010):144-155.

17. Endo D, Okada Y, Nagatani K, et al. Path following control for tracked vehicles based on slipcompensating odometry. Ieee/rsj International Conference on Intelligent Robots and Systems (2007):2871-2876 